\title{
Clinical Observation of Treating Infantile Ophthalmic Hemangioma by Oral Propranolol
}

\author{
Jing Huang*, Daoman Xiang \\ Guangzhou Women and Children's Medical Center, Guangzhou, China \\ Email address: \\ 331074139@qq.com (Jing Huang) \\ ${ }^{*}$ Corresponding author
}

To cite this article:

Jing Huang, Daoman Xiang. Clinical Observation of Treating Infantile Ophthalmic Hemangioma by Oral Propranolol. International Journal of Ophthalmology \& Visual Science. Vol. 4, No. 4, 2019, pp. 97-100. doi: 10.11648/j.ijovs.20190404.17

Received: October 1, 2019; Accepted: October 21, 2019; Published: November 19, 2019

\begin{abstract}
To evaluate the clinical efficacy and safety by oral propranolol in treating infantile ophthalmichemangioma (IH). A prospective analysis of 66 infantile outpatients with ophthalmic hemangioma from Feb 2016 to Apr 2018. According to the initial dose, the order of maintenance dose and drug withdrawal of oral propranolol prescription, the tumor size, color change and adverse reactions were studied and analyzed, and the clinical efficacy and safety were evaluated. The tumors became weak within 1 week in $83.3 \%$ outpatients (55 cases). 4 (6.1\%) outpatients received Grade I efficacy (ineffective), 7 (10.6\%) Grade II (poor), 9 (13.6\%) Grade III (improvement) and 46 (69.7\%) Grade IV (cured). The average prescribed medication treatment time was 6.9 months. The curative effect of the long course groups was better than the short ones, and 4-12 months group was the best. No obvious side effect was detected except 1 diarrhea. There was no significant difference in curative effect between left and right eyes or sex $(\mathrm{P}>0.05)$. Propranolol was used in outpatients to treat $\mathrm{IH}$, the indications and contraindications were strictly controlled and the clinical efficacy and safety were relatively satisfactory.
\end{abstract}

Keywords: Propranolol, Infantile, Eye, Hemangioma, Angioma

\section{Introduction}

Capillary hemangioma is a kind of benign tumor common occurs in infants and young children, especially occurs at birth or shortly after birth, so it is also known as infantile hemhemangioma (IH). The incidence of $\mathrm{IH}$ is about $1 \sim 2 \%$ [1]. IH can happen in any part of the body. $60 \%$ of hemhemangioma appears at head, neck and face. There are 3 phases: proliferation, rest and retrogression stage [8]. It can result blepharoptosis, exophthalmos and strabismus when IH arises at eyelid or orbit which may also affect the development of visual faction in time. Therefore, for the treatment of $\mathrm{IH}$, most scholars abandon the negative observation and follow-up strategy and advocate active intervention treatment. The commonly used treatments for IH include general use or local injection of glucocorticoids, pingyangmycin injection, cryotherapy, laser and surgery, with obvious side effects. Since 2008, many case reports and multicenter studies have confirmed that propranolol is safe and effective in the treatment of $\mathrm{IH}$ and has gradually replaced other methods as a first-line drug $[2,9-10,11]$. Oral propranolol treatment of $\mathrm{IH}$ requires hospitalization, but recent studies have reported that outpatient treatment is safe equally [2]. We got propranolol treatment used in outpatient in our hospital and its efficacy and safety were observed in detail.

\section{Subjects and Methods}

66 Participants with infantile ophthalmic hemhemangioma from Guangzhou Women and Children's Medical Center during February 2016 to April 2018 were enrolled in this study, aged 1 72 months, with an average of $10.53 \pm 15.09$ months. There were 20 males, 46 females, 35 right eyes and 31 left eyes. 4 cases were complicated with hemangioma in other parts of the body. There were 45 cases in proliferation stage ( 6 months), 3 cases in rest stage (6-12 months) and 18 cases in regression stage ( 1 year old $\sim$ ).

Selection criteria: 1) Children over 1 month old; 2) Ophthalmic hemangioma affected the appearance or caused dysfunction, including skin color changes, the tumor body protruded above the skin surface, ptosis, the eyeball displaced or the hemangioma ulcers; 3 ) The hemangioma of other parts 
of the whole body is combined.

Exclusion criteria: The contraindication listed for propranolol including heart disease (such as bradycardia, II-III AV block, heart failure), increased airway sensitivity (e.g. bronchial asthma), developmental abnormalities of the vascular or nervous system (such as the PHACES syndrome and the PELVIS syndrome), thyroid, liver or kidney disfunction.

\section{Study Methods}

1. Preparation for treatment: the medical history and family history were asked in detail to eliminate the contraindication of propranolol. The body weight and vital signs (body temperature, respiration, pulse, blood pressure) of the child were measured. Necessary inspection such as ultrasonic, MRI or CT examination was used to measure the size of the tumor. Electrocardiogram and heart color Doppler ultrasound were need to evaluate the cardiovascular system. The study requires the consent of the family.

2. Treatment: Propranolol for outpatient was $0.5 \sim 1 \mathrm{mg} / \mathrm{Kg}$.d, taken orally for $2 \sim 3$ times a day. The starting dose was adjusted according to the age. 0.5 $\mathrm{mg} / \mathrm{Kg}$.d at 6 months of age and $1 \mathrm{mg} / \mathrm{Kg}$.d after 6 months of age. The parents are told to closely observe the mental state of the children after taking the medicine and timely review when abnormal symptoms happened. It was planned to increase the dose to $1.5 \sim 2 \mathrm{mg} / \mathrm{Kg}$.d after 1 week for 2 3 times a day if there's no obvious abnormal reaction (such as dyspnea, slow reaction, listlessness, cold and numbness of the limbs, diarrhea, nausea, etc.) and maintain that therapeutic dose. The patients were checked every 2 weeks within 1 month, followed by a 4 -week review, and the dose was adjusted by body weight.

3. Review: The mental state, vital signs, the tumor size, color, tension, dysfunction, ulceration or tumor bleeding after taking propranolol were recorded. The imaging examination was used to assess the clinical efficacy and safety.

4. Evaluation criteria for efficacy: Grade I (ineffective): the tumor volume is continuously increasing or the treatment is stopped for some reason; Grade II (poor): the tumor volume is reduced by $25 \%$ or the color of tumor is lightened than before; Grade III (improvement): the tumor volume is reduced by $25-75 \%$ or the color of tumor is obviously lightened than before; Grade IV (cured): the tumor volume is reduced by more than $75 \%$ or the color of tumor is fading.

5. Indication and method of drug withdrawal: Enter the regression period and the tumor body is stable for more than 4 weeks or the tumor disappears. The dose was gradually reduced to $1 \mathrm{mg} / \mathrm{kg}$.d for 15 days and 0.5 $\mathrm{mg} / \mathrm{kg}$.d for 15 days. Other treatment methods should be used for the withdrawal in the case of Grade I effect for 1-3 months of treatment.

\section{Results}

1. In the total 66 cases, 3 cases were ineffective and transferred to interventional department for continuous treatment, and 1 case was stopped for some reason. In children with systemic multiple hemangioma, 1 case was treated with polidocanol sclerosis local injection and 1 case was treated with skin pulse dye laser. At the time of statistical analysis, the patients were taking medicine according to order.

2. The basic information of IH patients showed in Table 1. The color of tumor became lighter or tension decreased in 55 cases $(83.3 \%)$ with effect Grade I 4 cases $(6.1 \%)$, Grade II 7 cases (10.6\%), Grade III 9 cases (13.6\%) and Grade IV 46 cases $(69.7 \%)$ respectively. There was no significant difference in curative effect between left and right eyes or sex $(\mathrm{P}>0.05)$. The effect of the long course group was better than the short one, and the most effective course group was $4 \sim 12$ months, see Table 2 . The average treatment time was 6.9 months.

Table 1. Basic information of IH patients.

\begin{tabular}{|c|c|c|c|c|c|c|}
\hline & \multicolumn{3}{|l|}{ location } & \multicolumn{3}{|c|}{ Previous other treatment } \\
\hline & Right-eye & Left-eye & Intraorbit-al & general multiple & Methylpredniso-lone & propranolol \\
\hline case & 35 & 31 & 1 & 4 & 1 & 1 \\
\hline
\end{tabular}

Table 2. Effect of oral propranolol for IH and time relationship case number (\%).

\begin{tabular}{|c|c|c|c|c|c|}
\hline Treatment time (month) & case & I & II & III & IV \\
\hline 1 & 9 & $1(11.1)$ & $3(33.3)$ & $3(33.3)$ & $2(22.2)$ \\
\hline 2 & 9 & $1(11.1)$ & $2(22.2)$ & $2(22.2)$ & $4(44.4)$ \\
\hline 3 & 9 & $2(22.2)$ & $1(11.1)$ & $2(22.2)$ & $4(44.4)$ \\
\hline $4-6$ & 12 & 0 & 0 & $1(8.3)$ & $11(91.7)$ \\
\hline $7-9$ & 11 & 0 & $1(9.1)$ & 0 & $10(90.9)$ \\
\hline $10-12$ & 7 & 0 & 0 & 0 & $7(100)$ \\
\hline $13 \sim$ & 9 & 0 & 0 & $1(11.1)$ & $8(88.9)$ \\
\hline Total & 66 & $4(6.1)$ & $7(10.6)$ & $9(13.6)$ & $46(69.7)$ \\
\hline
\end{tabular}

With increase of the age when began oral propranolol, the possibility of reaching grade IV decreased gradually, as shown in figure 1 . 


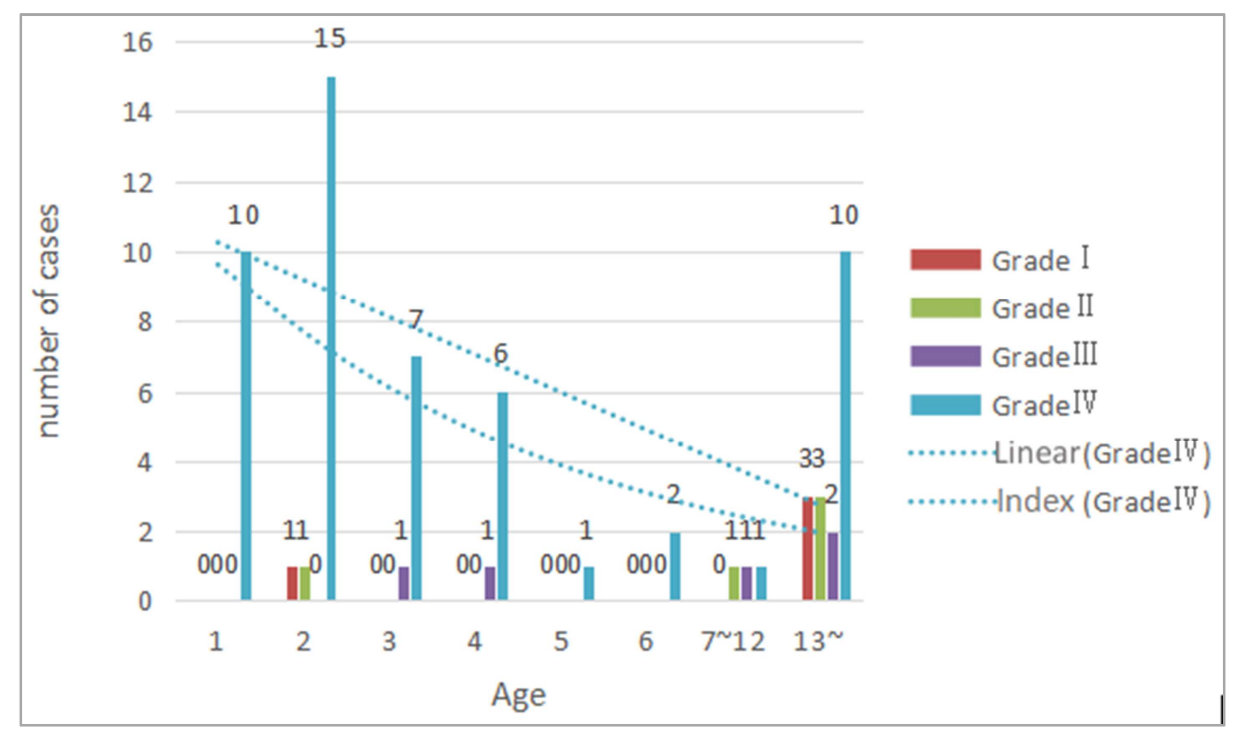

Figure 1. Relationship between age (month) of initiation of medication and efficacy.

Adverse reaction: A 2-months old patient had diarrhea after taken pills, the condition was improved after given symptomatic treatment and keep using the drug until 12 months for cure. The remaining children had no obvious side effects.

\section{Discussion}

$\mathrm{IH}$ is a very common benign tumor in infant, although it has 3 phases of proliferation, rest and retrogression stage, when the hemangioma occurs on the face, especially the eye, it urges the parents to go to the doctor because of the influence of beauty or the possibility of decreasing vision. Pingyangmycin injection for the ophthalmic hemangioma as a prior treatment is good but some of the children may have scar or skin necrosis [3]. One case of obstruction type hypertrophic cardiomyopathy combined with nasal hemangioma was found that the tumor was obviously improved when treated with 3 $\mathrm{mg} / \mathrm{kg}$.d propranolol in 2008, then $2 \mathrm{mg} / \mathrm{kg}$.d propranolol for the other 10 cases of hemangiomas obtained good effects. A large number of cases since then were studied in the whole world, propranolol as a first-line drug in the treatment of $\mathrm{IH}$ had not been found serious side effects [2, 9-11], even the outpatient treatment was safe and effective [2], so be hospitalized was not required in our hospital. The medical history and the necessary examinations were inquired before the oral propranolol treatment, and the contraindications of the medicine were eliminated. In this study, there were no obvious side effects except 1 case had diarrhea and disappeared after the symptomatic treatment.

In our study, $83.3 \%$ of the children had got lighter color or tension decreased of the tumor within 1 week. The younger the child was when the drug was started, the more likely he was to be cured, especially in the 4-12 months group. One 24-months-old child had a relapse after 1 year of drug withdrawal, and was cured after 6 months of treatment with a second dose of the drug. Propranolol is a non-selective $\beta$-adrenergic receptor blocker, and $\beta 1$ and $\beta 2$ receptors acts on cell membranes may cure IH through three molecular mechanisms as below: promoting the vasoconstriction, inhibiting the angiogenesis and inducing the apoptosis of the endothelial cells [4]. The tumor became lighter in color, which can be observed more often 1 to 3 days after administration by constricting the blood vessels and reducing blood flow. Mitogen activates the protein kinase pathway and reduces the expression of $\mathrm{bFGF}$ and VEGF to inhibit angiogenesis. The levels of bFGF and VEGF in the proliferating phase of hemangioma were significantly higher than those in the regression phase of hemangioma [4-5]. Apoptosis is one of the important causes of spontaneous regression of hemangioma. The expression of apoptosis signals such as bcl-2, bax, Fas and caspase-3 in hemangioma in the regression stage was significantly higher than that in the proliferation stage. Pronaprol induced an increase in apoptosis by blocking the beta 2 receptor involved in the signaling pathway of apoptosis [6]. Some studies have pointed out that, in order to avoid the rebound growth of hemangioma, the optimal treatment time should cover most or even all of the proliferating stage, at least until the children are 7-8 months old or the tumor enters the regression stage and stable more than 4 weeks [7].

Among the children with effect Grade I, 3 cases were diagnosed separately with left upper eyelid vascular malformation, left intraorbital venous hemangioma and right subconjunctival vasculogenic lesions on MRI, and were transferred for interventional treatment in time to avoid delay in treatment. One 21-months-old child had a poor effect by methylprednisolone for 1 year in another hospital, and then got effect Grade II after propranolol for 1 month, so we stopped the drug because of the regression stage coming. The tumor was misdiagnosed as a megalomyelomian gland cyst which on the conjunctival surface of the eyelid presented a purply-red round shape and other dacryocyst mass with overflow was misdiagnosed as dacryocystitis, suggesting that we should pay attention to the differential diagnosis and necessary imaging examination. 


\section{Conclusion}

Propranolol was used in outpatients to treat IH, the indications and contraindications were strictly controlled and the clinical efficacy and safety were relatively satisfactory. However, due to the mechanism of action, optimal dose range, treatment cycle and long-term follow-up results of the drug were not very accurate, further exploration and research were needed in our clinical work.

\section{References}

[1] Fengming Li. Chinese Ophthalmology. Version 2. Beijing: People's Health Press, 2005.

[2] Haider K, Plager DA, Neely DE, et al. Outpatient treatment of periocular infantile hemhemangiomas with oral propranlolo. J AAPOS. 2010, 14: 251-256.

[3] Daoman Xiang, Hongmei Zhou, Guangqiang Feng, et al. Chinese Journal of Medical Aesthetics and Cosmetology. 2006, 12 (2): 71-73.

[4] Przewratil P, Sitkiewicz A, Wyka K, et al. Serum levels of vascular endothelial growth factor and basic fibroblast growth factor in children with hemhemangiomas and vascular malformations preliminary report. Pediatr Dematol, 2009, 26: 399-404.

[5] Przewratil P, Sitkiewicz A, Andrzeijwska E. Local serum levels of vascular endothelial growth factor in infantile hemhemangioma: intriguing mechanisms of endothelial growth. Cytokine, 2010, 49: 141-147.

[6] Ming Zhang, Qiang Peng, Ming Liu. Expression of NF-kappa B in propranolol treatment for infantile hemangioma in nude mice model. Journal of Clinical Pediatric Surgery. 2019, 18 (8): 655-659.

[7] Holmes WJ, Mishra A, Gorst C, et al. Propranolol as first-line treatment for rapidly proliferating Infantile Haemhemangiomas. J Plast Reconstr Aesthet Surg, 2011, 64: 445-451.
[8] Tingting Lin, Yanjin He. The advance of $\beta$-blockers in the treatment of infantile hemangiomas. Chinese Journal of Ophthalmology. 2013, 49 (12): 1138-1144.

[9] Lu Yu, Li Li, Lin Ma. Short-and long-term safety of oral propranolol in the treatment of infantile hemangioma.

[10] Chinese Journal of Dermatology. 2019, 52 (8): 586-589.

[11] Jianmei Huang, Wenying Liu. A literature review of treating infantile hemangiomas with propranolol. Chinese Journal of Pediatric Surgery. 2018, 39 (2): 150-154.

[12] Yanhui Cui, Qian Wu, Dayong Bai, et al. Clinical observation for treating children eyelid periorbital hemangioma by oral propranolol. Chinese Journal of Strabismus \& Pediatric Ophthalmology. 2015, 23 (1): 18-23.

[13] The Chinese version of this article was published in the journal of clinical ophthalmology in June 2019.

\section{Biography}

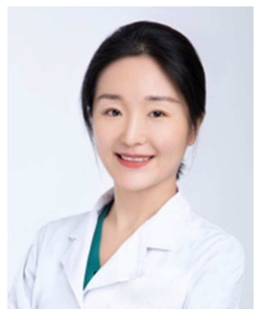

Jing Huang, Graduated from Shantou University Medical College with a maste degree in myopia and Pediatric Ophthalmology. Have extensive clinical experience in dealing with common ophthalmic diseases in adults and children, especially good at ametropia correction and treatment or operation for pediatric eye

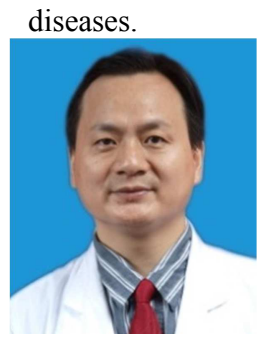

Daoman Xiang, Doctor, chief physician, master tutor. Specialized in fundus screening of premature infants, strabismus and amblyopia in children, various eye tumors and congenital eye diseases. 